\title{
Precedence tests and Lehmann alternatives
}

\author{
P. van der Laan \\ Department of Mathematics and Computing Science \\ Eindhoven University of Technology \\ Eindhoven, The Netherlands \\ S. Chakraborti \\ Department of Management Science and Statistics \\ University of Alabama \\ Tuscaloosa, U.S.A.
}

Key Words: Best precedence tests; nonparametric; power; proportional hazards alternatives; semi-parametric.

AMS Classification: Primary 62G10; Secondary 62G25, 62G30.

Support provided in part by NATO Collaborative Research Grant CRG 920287. Research partially supported by European Union HCM Grant ERB CHRX-CT940693. 


\section{Abstract}

Precedence tests are considered in the context of four classes of one-sided Lehmann-type alternatives: $G=F^{k}(k>1) ; G=1-(1-F)^{k}(k<1) ; G=$ $F^{k}(k<1)$; and $G=1-(1-F)^{k}(k>1)$, where $F$ and $G$ are two continuous cumulative distribution functions. If an optimal precedence test (one with the maximal power) is determined for one of these four classes, the optimal tests for the other classes of alternatives can be derived. Application of this is given using the results of Lin and Sukhatme (1992) who derived the best precedence test for testing the null hypothesis that the lifetimes of two types of items on test have the same distribution. The test has maximum power for fixed $k$ in the class of alternatives $G=1-(1-F)^{k}$ with $k<1$. Best precedence tests for the other three classes of Lehmann-type alternatives are derived using their results.

\section{Introduction}

The one-sided two-sample problem is one of the basic problems in statistical testing. Assume that two independent samples $X_{1}, \ldots, X_{m}$ and $Y_{1}, \ldots, Y_{n}$ from continuous cumulative distribution functions $F$ and $G$, respectively, are given. We wish to test the null hypothesis $H_{0}: F(x)=G(x)$ for all $x$ against the alternative hypothesis $H_{a}: G(x) \leq F(x)$ for all $x$ with strict inequality sign for at least one value of $x$. Many nonparametric tests for the two-sample problem are considered in the literature. In this paper only precedence tests are considered. General description and properties of precedence tests, as well as an overview of the literature are given in Chakraborti and van der Laan (1996 and 1997) for complete data and for censored data, respectively. In section 2 a short overview of Lehmann alternatives and proportional hazards alternatives is given. The power of precedence tests is given in section 3 . In section 4 it is proved that the power of a precedence test against four classes of Lehmann alternatives or proportional hazards alternatives can be derived if the power against one class of alternatives is given. 


\section{Some classes of Lehmann-type alternatives}

If, in general, we wish to test the null hypothesis $H_{0}: F(x)=G(x)$ for all $x$ in the two-sample problem, alternatives that are considered often are the shift alternatives. Nonparametric tests are compared with each other, and with their parametric counterparts by means of power against shift alternatives. We are thus led to examine the power of nonparametric tests. One possibility is to test against parametric alternatives as shift alternatives. Then the power of a nonparametric test depends on the form of the underlying distribution. So we can compute power, for example, under normal distribution theory. There is nothing contradictory about this because we examine power in order to determine how much we can stand to loose in power by using a nonparametric test, compared with its classical counterpart, if the normal theory assumptions are really valid. However, for many distribution functions the computation of the power function of many nonparametric tests has been a mathematically difficult problem. Partly to alleviate this problem Lehmann (1953) introduced and proposed to use the so-called Lehmann alternatives in power calculations of two-sample rank tests.

Suppose the order statistics of the $X$-observations are denoted by $X_{(1)}<$ $\ldots<X_{(m)}$ and the order statistics of the $Y$-observations by $Y_{(1)}<\ldots<Y_{(n)}$. The ranks of the $X$ 's and $Y$ 's in the combined sample are denoted by $R_{1}<\ldots<R_{m}$ and $S_{1}<\ldots<S_{n}$, respectively. According to Lehmann (1953) suppose that $F$ and $G$ are related as $G=g(F)$, where $g$ is a continuous non-decreasing function differentiable on $[0,1]$ with derivative $g^{\prime}$. The function $g()=.G F^{-1}($.$) is sometimes referred to as the "conversion" function.$ Then Lehmann (1953) showed that

$$
P\left(S_{1}=s_{1}, \ldots, S_{n}=s_{n}\right)=\left(\begin{array}{c}
N \\
m
\end{array}\right)^{-1} \mathcal{E}\left\{g^{\prime}\left(U^{\left(s_{1}\right)}\right) \ldots . g^{\prime}\left(U^{\left(s_{n}\right)}\right)\right\},
$$

where $U^{\left(s_{1}\right)}, \ldots, U^{\left(s_{n}\right)}$ are the $s_{1}$-st to $s_{n}$-th order statistics in a sample of $N=m+n$ variables distributed uniformly on $[0,1]$. From this it can be deduced that the difficulties in obtaining power results for a specific alternative is related to the complexity of $g$ involved. For instance, for normal shift alternatives $F(x)=\Phi(x)$ and $G(x)=\Phi(x-\delta)$, the conversion function $g=G\left(F^{-1}\right)$ is not easy to handle. Considering the one-sided alternative $H_{a}: G(x)<F(x)$ for all $x$, the simplest choice seems to be $g(x)=x^{k}, k>1$. This corresponds with the Lehmann alternative $H_{a}: G(x)=F^{k}(x), k>1$. 
Further, as a practical motivation, note that, for a positive integer $k, G=F^{k}$ is the cumulative distribution function of the maximum of $k$ independently distributed random variables with common cumulative distribution function $F(x)$ and $1-\{1-F(x)\}^{k}$ is the cumulative distribution function of the minimum of $k$ independently distributed random variables with common cumulative distribution function $F(x)$. These alternatives, often referred to as semi-parametric alternatives, are of practical interest in life testing, reliability testing, extreme-value distribution investigations and related fields.

Note that for the extreme-value distribution

$$
F(x)=\exp \left(-e^{-x / \beta}\right) \quad(\beta>0 ;-\infty<x<\infty)
$$

the Lehmann alternative

$$
F^{k}(x)=\exp \left(-k e^{-x / \beta}\right)
$$

is equivalent to a shift alternative

$$
F(x-\delta)=\exp \left(-e^{-(x-\delta) / \beta}\right),
$$

where

$$
k=e^{\delta / \beta} \text { or } \delta=\beta \ln k .
$$

In the case of $g(x)=x^{k}$, which corresponds to $G=F^{k}$, Lehmann (1953) obtained from (1) that

$$
P\left(S_{1}=s_{1}, \ldots, S_{n}=s_{n}\right)=k^{n}\left(\begin{array}{l}
N \\
m
\end{array}\right)^{-1} \sum_{j=1}^{n} \frac{\Gamma\left(s_{j}+j k-j\right) \Gamma\left(s_{j+1}\right)}{\Gamma\left(s_{j}\right) \Gamma\left(s_{j+1}+j k-j\right)} .
$$

So, the power of various rank tests can be computed simply by summing up the right-hand side of $(6)$ over all sets $\left(s_{1}, \ldots, s_{n}\right)$ which are elements of the critical region.

More generally, one can discern four classes of Lehmann-type alternatives for positive $k$ :

1. $H_{1}: G(x)=F^{k}(x)$ for all $x$ and $k>1$, which for small $k$ can be considered as a rough approximation for a shift of $G($.$) to the right$ with respect to $F($.$) ;$ 
2. $H_{2}: G(x)=1-\{1-F(x)\}^{k}$ for all $x$ and $k<1$, which for $k$ not too far from 1 , can be considered as a rough approximation for a shift of $G($.$) to the right with respect to F($.$) ;$

3. $H_{3}: G(x)=F^{k}(x)$ for all $x$ and $k<1$, which for $k$ not too far from 1 , can be considered as a rough approximation for a shift of $G($.$) to the$ left with respect to $F($.$) ;$

4. $H_{4}: G(x)=1-\{1-F(x)\}^{k}$ for all $x$ and $k>1$, which for small $k$ can be considered as a rough approximation for a shift of $G($.$) to the left$ with respect to $F($.$) .$

The alternatives $H_{1}$ and $H_{3}$ are known as Lehmann alternatives, whereas $H_{2}$ and $H_{4}$ are more commonly known under the name of proportional hazards alternatives. This is since under $\mathrm{H}_{2}$ and $\mathrm{H}_{4}$, the ratio of the two hazard functions, defined as $[f(x) /\{1-F(x)\}] /[g(x) /\{1-G(x)\}]$, is a constant. It seems that where nonparametric tests are appropriate one usually does not have a very precise knowledge of the alternatives and in those situations these Lehmann-type (semi-parametric) alternatives provide a good choice.

\section{The power of a precedence test}

Let $V_{i}$ denote the total number of $Y$-observations that do not exceed $X_{(i)}$, i.e. preceed $X_{(i)}(i=1, \ldots, m)$. The statistic $V_{i}$ is called a precedence statistic and a test based on $V_{i}$ is referred to as a precedence test. Testing $H_{0}: F(x) \equiv G(x)$ against $H_{a}: G(x)<F(x)$ using a precedence test the null hypothesis will be rejected if and only if $V_{i}<j$, where $j$ has to be determined such that the size of the test is smaller then or equal to a predetermined value $\alpha(0<\alpha<1)$. A test based on the precedence statistic $V_{i}$ is statistically equivalent to a test based on two order statistics, one from each sample. This follows since

$$
V_{i}<j \Leftrightarrow n G_{n}\left(X_{(i)}\right)<j \Leftrightarrow Y_{(j)}>X_{(i)}
$$

where $G_{n}($.$) is the empirical distribution function of the Y$-sample. When data become available in increasing order of magnitude, such as in life testing, 
a decision can be reached and hence experimentation can be stopped as soon as either $X_{(i)}$ or $Y_{(j)}$ is observed, whichever came first. If $X_{(i)}$ is observed before $Y_{(j)}$ then $H_{0}$ is rejected, whereas if $Y_{(j)}$ is observed before $X_{(i)}$, then $H_{0}$ is retained. The early termination is an attractive feature of a precedence test because of the potential savings in time and resources.

For the power of a precedence test we get, in general,

$$
\begin{aligned}
\beta(F, G) & =P\left(Y_{(j)}>X_{(i)}\right) \\
& =P\left(V_{i} \leq j-1\right) .
\end{aligned}
$$

It can be shown (see for example Chakraborti and van der Laan (1996)) that

$$
\begin{aligned}
P\left(V_{i}=s\right)= & \left(\begin{array}{l}
n \\
s
\end{array}\right) B^{-1}(i, m-i+1) \\
& \int_{0}^{1}\left\{G F^{-1}(u)\right\}^{s}\left\{1-G F^{-1}(u)\right\}^{n-s} u^{i-1}(1-u)^{m-i} d u .
\end{aligned}
$$

So the power is

$$
\beta(F, G)=\sum_{s=0}^{j-1} P\left(V_{i}=s\right),
$$

where $j$ is the largest integer for which $\beta(F, F) \leq \alpha$ with $0<\alpha<1$. When $F=G,(8)$ simplifies to

$$
P\left(Y_{(j)}>X_{(i)}\right)=\sum_{s=0}^{j-1}\left(\begin{array}{c}
i+s-1 \\
s
\end{array}\right)\left(\begin{array}{c}
N-i-s \\
n-s
\end{array}\right)\left(\begin{array}{c}
N \\
n
\end{array}\right)^{-1} .
$$

From (8) it is again clear that the difficulty to determine the power of a precedence test depends on the complexity of the conversion function $g=$ $G F^{-1}$. However, if we use Lehmann-type alternatives, the power of a precedence test can be computed explicitly. 
In this case we get from (9) and (10)

$$
\begin{aligned}
\beta\left(F, F^{k}\right)= & \sum_{s=0}^{j-1}\left(\begin{array}{l}
n \\
s
\end{array}\right) B^{-1}(i, m-i+1) \\
& \int_{0}^{1} u^{k s+i-1}(1-u)^{m-i}\left(1-u^{k}\right)^{n-s} d u \\
= & \sum_{s=0}^{j-1}\left(\begin{array}{l}
n \\
s
\end{array}\right) B^{-1}(i, m-i+1) \\
& \sum_{r=0}^{n-s}(-1)^{r}\left(\begin{array}{c}
n-s \\
r
\end{array}\right) B(k(r+s)+i, m-i+1) \\
= & \sum_{s=0}^{j-1} \sum_{r=0}^{n-s}(-1)^{n-r-s} C_{r, s}^{n}\left[\prod_{l=i}^{m}\left\{1+\frac{k}{l}(n-r)\right\}\right]^{-1},
\end{aligned}
$$

with $C_{r, s}^{n}=\frac{n !}{r ! s !(n-r-s) !}$.

In a similar way the power of a precedence test can be determined for the proportional hazards alternatives. We get

$$
\begin{aligned}
\beta\left(F, 1-(1-F)^{k}\right)= & \sum_{s=0}^{j-1}\left(\begin{array}{l}
n \\
s
\end{array}\right) B^{-1}(i, m-i+1) \\
& \int_{0}^{1} u^{i-1}(1-u)^{m-i+k(n-s)}\left\{1-(1-u)^{k}\right\}^{s} d u \\
& =\sum_{s=0}^{j-1}\left(\begin{array}{l}
n \\
s
\end{array}\right) B^{-1}(i, m-i+1) \\
& =\sum_{s=0}^{j-1} \sum_{r=0}^{s}(-1)^{r} C_{r, s-r}^{n}(-1)^{r}\left(\begin{array}{l}
s \\
r
\end{array}\right) B(i, m-i+1+k(n+r-s)) \\
& {\left[\prod_{l=m}^{m}\left\{1+\frac{k}{l}(n+r-s)\right\}\right]^{-1} . }
\end{aligned}
$$


In the next section certain relations between the power functions for the Lehmann-type alternatives are given. From these relations optimal precedence tests can easily be determined for all Lehmann-type alternatives if the optimal precedence test for one class is given.

\section{Relations between optimal precedence tests}

Assume that an optimal (i.e. has largest power) one-sided precedence test against $H_{2}$ is given as in Lin and Sukhatme (1992). Such a test rejects $H_{0}$ if and only if $Y_{(j)}-X_{(i)}>0$. Then the corresponding one-sided precedence test, testing against the alternative hypothesis $H_{3}$, rejects $H_{0}$ if and only if $Y_{(s)}-X_{(r)}<0$ for certain values of $r$ and $s$. The following relation can be used in order to find $r$ and $s$, when $i$ and $j$ are given (and also the optimality follows):

$$
\begin{aligned}
P\left(Y_{(j)}\right. & \left.\left.>X_{(i)}\right) \mid G=1-(1-F)^{k}, k<1\right) \\
& =P\left(V_{i} \leq j-1 \mid 1-G=(1-F)^{k}, k<1\right) \\
& =P\left(\sharp\left\{X<Y_{(j)}\right\} \geq i \mid 1-G=(1-F)^{k}, k<1\right) \\
& =P\left(Y_{(n-j+1)}<X_{(m-i+1)} \mid G=F^{k}, k<1\right) .
\end{aligned}
$$

In the appendix an algebraic proof is also given. From the result given in (12) the next Lemma follows:

Lemma 1 If $Y_{(j)}-X_{(i)}$ is the best precedence test statistic for $H_{2}$, rejecting $H_{0}$ when the test statistic is positive, then $Y_{(n-j+1)}-X_{(m-i+1)}$ is the best precedence test statistic for $H_{3}$, rejecting $H_{0}$ if and only if it is negative.

In a similar way Lemma 2 follows:

Lemma 2 If $Y_{(j)}-X_{(i)}$ is the best precedence test statistic for $H_{4}$, rejecting $H_{0}$ if it is negative, then $Y_{(n-j+1)}-X_{(m-i+1)}$ is the best precedence test statistic for $H_{1}$, rejecting $H_{0}$ if and only if it is positive. 


\section{Application 1}

Lin and Sukhatme (1992) gives the best precedence test for $H_{2}$. For instance, if $k=1 / 2, \alpha=.05, m=10$ and $n=8$, then from Lin and Sukhatme (1992; Table II) it follows that the best (nonrandomized) precedence test based on the statistic $Y_{(5)}-X_{(10)}$, rejecting $H_{0}$ if and only if $Y_{(5)}-X_{(10)}>0$, is the best (maximal power) precedence test for testing $H_{0}$ against $H_{2}$. From Lemma 1 it follows that the precedence test based on the statistic $Y_{(4)}-X_{(1)}$, rejecting $H_{0}$ if and only if $Y_{(4)}-X_{(1)}<0$, is the best precedence test for testing $H_{0}$ against $\mathrm{H}_{3}$.

Next, starting from $H_{3}$ we can find the best precedence test against $H_{1}$ as follows. We have

$$
P\left(Y_{(j)}<X_{(i)} \mid G=F^{k}, k<1\right)=P\left(X_{(i)}>Y_{(j)} \mid F=G^{k^{*}}, k^{*}>1\right),
$$

with $k^{*}=k^{-1}$. From this Lemma 3 follows.

Lemma 3 If for reversing sample sizes $n$ (for the $X$-sample) and $m$ (for the $Y$-sample) the test based on the precedence test statistic $Y_{(j)}-X_{(i)}$, rejecting $H_{0}$ if and only if $Y_{(j)}-X_{(i)}<0$, is the best precedence test against $H_{3}$ with $k=k_{0}$, then the test based on $Y_{(i)}-X_{(j)}$, rejecting $H_{0}$ if and only if $Y_{(i)}-X_{(j)}>0$, is the best precedence test for testing $H_{0}$ against $H_{1}$ with $k=$ $k_{0}^{-1}$ and sample sizes $m$ and $n$ for the $X$-sample and $Y$-sample, respectively.

\section{Application 2}

Suppose the best precedence test is desired against $H_{1}$ for $m=8, n=12$ and $k=2$. According to Lemma 3 we first find the best test against $H_{3}$ for $m=12, n=8$ and $k=1 / 2$. From Lin and Sukhatme(1992; Table II) and using Lemma 1 the best (nonrandomized) precedence test, at $\alpha=0.05$ is $Y_{(3)}-X_{(1)}<0$. Then by Lemma 3 , the best precedence test for the given problem is simply $Y_{(1)}-X_{(3)}>0$.

Finally, starting from $\mathrm{H}_{2}$ one can also find the best precedence test against $\mathrm{H}_{4}$ as follows. We have

$$
\begin{aligned}
P\left(Y_{(j)}>X_{(i)} \mid G\right. & \left.=1-(1-F)^{k}, k<1\right) \\
& =P\left(X_{(i)}<Y_{(j)} \mid F=1-(1-G)^{k^{*}}, k^{*}>1,\right)
\end{aligned}
$$


with $k^{*}=k^{-1}$. From this equality the next Lemma follows.

Lemma 4 The best precedence test for testing $H_{0}$ against $H_{4}$ with $k=k_{0}$ and sample sizes $m$ and $n$, and rejecting $H_{0}$ if and only if $Y_{(j)}-X_{(i)}<0$, can be found by taking the best precedence test against $H_{2}$ with $k=k_{0}^{-1}$, rejecting $H_{0}$ if and only if $Y_{(i)}-X_{(j)}>0$ and reversing sample sizes $n$ and $m$.

\section{Application 3}

Suppose we need the best precedence test against $H_{1}$ for $m=8, n=6$ $k=1 / 2$ and $\alpha=0.05$. According to the Lemma we first find the best (nonrandomized) test against $H_{2}$ for $m=6, n=8$ and $k=2$. From Lin and Sukhatme(1992; Table II) this is , at $\alpha=0.05, Y_{(4)}-X_{(6)}>0$. So the required best precedence test is simply $Y_{(6)}-X_{(4)}<0$.

\section{Remark}

Starting from the best precedence test against $H_{2}$, and using the results of the four Lemma's, the best precedence test against $H_{3}$ (Lemma 1 ) and $H_{4}$ (Lemma 4 ) can be found and thus also against $H_{1}$ (Lemma's 2 and 3 ).

\section{Appendix}

$$
\begin{aligned}
p_{1}:= & P\left(Y_{(j)}-X_{(i)}>0 \mid G=1-(1-F)^{k}, k<1\right) \\
& =\sum_{s=0}^{j-1} P\left(V_{i}=s \mid G=1-(1-F)^{k}, k<1\right) \\
& =\sum_{s=0}^{j-1}\left(\begin{array}{l}
n \\
s
\end{array}\right) B^{-1}(i, m-i+1) \\
& \sum_{r=0}^{s}(-1)^{r}\left(\begin{array}{l}
s \\
r
\end{array}\right) B(i, m-i+1+k(n+r-s)),
\end{aligned}
$$


whereas

$$
\begin{aligned}
p_{2}: & =P\left(Y_{(n-j+1)}-X_{(m-i+1)}<0 \mid G=F^{k}, k<1\right) \\
& =P\left(V_{m-i+1} \geq n-j+1 \mid G=F^{k}, k<1\right) \\
& =\sum_{s=n-j+1}^{n} P\left(V_{m-i+1}=s \mid G=F^{k}, k<1\right) \\
& =\sum_{s=n-j+1}^{n}\left(\begin{array}{c}
n \\
s
\end{array}\right) B^{-1}(m-i+1, i) \\
& \sum_{r=0}^{n-s}(-1)^{r}\left(\begin{array}{c}
n-s \\
r
\end{array}\right) B(m-i+1+k(r+s), i),
\end{aligned}
$$

and the equality $p_{1}=p_{2}$ follows easily by transforming the summation variable $s$ to $n-t$.

\section{References}

Chakraborti, S. and van der Laan, P. (1996). Precedence tests and confidence bounds for complete data: An overview and some results. The Statistician 45, 351-369.

Chakraborti, S. and van der Laan, P. (1997). An overview of precedence-type tests for censored data. Biometrical Journal 39, 99 - 116.

Lehmann, E.L. (1953). The power of rank tests. Ann. Math. Statist. 24, 23 -43 .

Lin, C. H. and Sukhatme, S. (1992). On the choice of precedence tests. Commun. Statist.-Theor. Meth. A21, $2949-2968$. 